\title{
Nucleation and growth kinetics of colloidal nanoparticles CdS:Mn in aqueous solution of polyvinyl alcohol
}

\author{
G.Yu. Rudko ${ }^{1}$, V.I. Fediv ${ }^{2}$, A.I. Savchuk ${ }^{3}$, E.G. Gule ${ }^{1}$, I.P. Vorona ${ }^{1}$, V.V. Nosenko ${ }^{1}$ \\ ${ }^{I} V$. Lashkaryov Institute of Semiconductor Physics, National Academy of Sciences of Ukraine, \\ 45, prospect Nauky,03028Kyiv, Ukraine, e-mail: g.yu.rudko@gmail.com \\ ${ }^{2}$ Bukovinian State Medical University, Department of Biophysics and Medical Informatics, \\ 42, Kobylianska str., 58000 Chernivtsi, Ukraine, \\ ${ }^{3}$ Chernivtsi National University, 2, Kotsyubynsky str., 58012 Chernivtsi, Ukraine
}

\begin{abstract}
Colloidal undoped CdS and doped CdS:Mn nanoparticles were synthesized in the solution of polyvinyl alcohol polymer, and the process of nanoparticles growth was monitored by optical absorption measurements. The inclusion of $\mathrm{Mn}^{2+}$ impurity was checked by electron paramagnetic resonance measurements. It is shown that the core of CdS:Mn nanoparticle is undoped. The results obtained demonstrated that the rate of the band gap value decrease during nanoparticles growth is slower for the Mn-doped nanoparticles as compared to the undoped ones. This effect was ascribed to the inhibition of nanoparticles growth in the solution containing manganese salt. The model of the adsorptive doping of CdS NPs with manganese was proposed.
\end{abstract}

Keywords: colloidal synthesis, nanoparticles, doping, CdS:Mn.

Manuscript received 28.03.14; revised version received 09.07.14; accepted for publication 16.09.14; published online 30.09.14.

\section{Introduction}

The insertion of just a few impurity atoms into a host semiconductor nanoparticle (NP) can drastically alter its phase, shape, and physical properties. The synthesis of doped nanomaterials with a specific dopant at a desired site in a host that leads to targeted physical properties requires fundamental understanding of the doping process. Colloidal doped NPs have drawn considerable attention in recent years because of their unique optical, electronic, and magnetoptical properties that stimulate potential applications in optoelectronic devices, biomedical labelling, etc. [1-4].

One particularly interesting and potentially beneficial approach is the synthesis of hybrid materials, consisting of inorganic doped NPs and organic polymers [5]. Such a combination may facilitate the application of unique properties of NPs by providing compatibility with existing techniques of polymers processing. Determination of the conditions and consistent patterns of the formation of crystal nuclei of adsorptionally/chemically alloyed NPs in aqueous polymeric solutions and studies of their physicochemical properties are a pressing problem of modern nanoelectronics. In these hybrids the role of highmolecular substances lies in controlling the size and the form of NPs, avoiding their aggregation and surface oxidation. Such nanostructures are used in the solar cells, biosensors, sources of light [6-9]. Understanding of how nanoparticles grow in various media is important in rationally designing synthetic routes to high quality composite colloids of desirable morphology and composition.

In the present study we used optical methods as one of the most available to study in the building of NPs and magnetic resonance methods to control the magnetic dopant incorporation. We first discuss the synthesis of polymer hybrid colloids containing either undoped or doped colloidal NPs in the solution of polyvinyl alcohol (PVA) and analyse how each step of synthesis influences 
the band gap of NPs. We then demonstrate the presence of the embedded impurity in colloidal NPs by EPR mearsurements. Finally, we propose a scheme of the doping of NPs with the magnetic impurity for the system (PVA- $\mathrm{H}_{2} \mathrm{O}-\mathrm{CdS}: \mathrm{Mn}$ nanoparticles).

\section{Synthesis details and characterization techniques}

Colloidal semiconducting NPs, both undoped CdS and CdS:Mn, were synthesized in the water solution of polyvinyl alcohol (PVA). The precursors for undoped $\mathrm{CdS}$ NPs $\left(\mathrm{Cd}^{2+}\right.$ and $\mathrm{S}^{2-}$ ions) were injected into the growth solution sequentially via step-wise additions of equal amounts of $\mathrm{CdCl}_{2}$ and $\mathrm{Na}_{2} \mathrm{~S}$ salts solutions. The presence of the capping molecules of polymer secured the restriction of NPs growth and formation of the stable colloid. The detailed sequence of the synthesis procedures is shown in Fig. 1.

To synthesize Mn-doped NPs the water solution of the salt $\mathrm{MnCl}_{2}$ was added to the initial growth solution of PVA before adding other precursors. Afterwards the growth was conducted by the same sequence of precursors additions as above. The detailed quantitative characteristics of the growth solution composition at different stages of the synthesis are listed in Table. All source chemicals were chemically pure. The concentration of salts solutions in all cases was $0.1 \mathrm{~mol} / \mathrm{l}$. The bi-distilled water was used for solutions preparation. The synthesis conditions $(\mathrm{pH}$ values and precursors concentrations) were adjusted to meet the conditions of colloid formation and to avoid precipitation of $\mathrm{MnS}, \quad \mathrm{Mn}(\mathrm{OH})_{2}$ and $\mathrm{Cd}(\mathrm{OH})_{2}$. Concentration of polymer in the initial growing solution was $5 \mathrm{wt} \%$, concentration of $\mathrm{Mn}^{2+}$ was $5 \cdot 10^{-3} \mathrm{M}$, concentration of $\mathrm{Cd}^{2+}$ ions varied in the range (1$30 \mathrm{mM}$ ). All synthesis steps were done at ambient conditions.

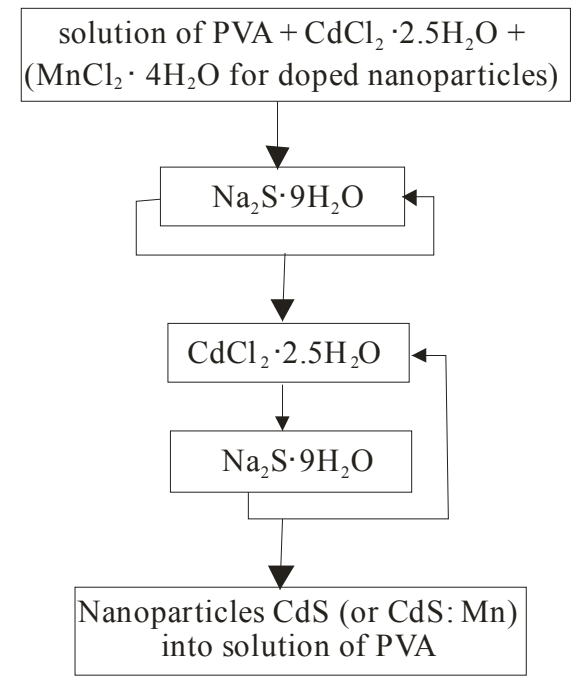

Fig. 1. The scheme of the synthesis of undoped CdS and CdS:Mn NPs in the water solution of polyvinyl alcohol.
Table. The sequence of the precursors injections into the reaction solution at different stages of synthesis of $\mathrm{CdS}$ and CdS:Mn NPs.

\begin{tabular}{|c|c|c|c|c|c|c|}
\hline \multicolumn{3}{|c|}{ CdS } & \multicolumn{4}{|c|}{ CdS:Mn } \\
\hline \multirow[t]{2}{*}{ Stage } & \multicolumn{2}{|c|}{$\begin{array}{c}\text { Concentration } \\
\text { of ions, } \\
10^{-3} \mathrm{~mol} / \mathrm{dm}^{3}\end{array}$} & \multirow[t]{2}{*}{ Stage } & \multicolumn{3}{|c|}{$\begin{array}{l}\text { Concentration of ions, } \\
10^{-3} \mathrm{~mol} / \mathrm{dm}^{3}\end{array}$} \\
\hline & $\mathrm{Cd}^{2+}$ & $\mathrm{S}^{2-}$ & & $\mathrm{Cd}^{2+}$ & $\mathrm{S}^{2-}$ & $\mathrm{Mn}^{2+}$ \\
\hline 1 & 1 & 0 & 1 & 1 & 0 & 2.5 \\
\hline 2 & 0.97 & 0.19 & 2 & 0.97 & 0.39 & \\
\hline 3 & 0.96 & 0.39 & 3 & 0.96 & 0.77 & \\
\hline 4 & 0.96 & 0.77 & 4 & 1.20 & 1.15 & \\
\hline 5 & 0.96 & 1.15 & 5 & 1.67 & 1.72 & \\
\hline 6 & 1.2 & 1.34 & 6 & 2.59 & 2.73 & \\
\hline 7 & 2.11 & 2.35 & & & & \\
\hline 8 & 3.03 & 3.36 & & & & \\
\hline
\end{tabular}

Absorption spectra were measured using MDR-24 grating monochromator at room temperature. The UVlamp was used as the light sources.

For EPR measurements the thin self-standing films of semiconductor nanoparticles/PVA nanocomposites were used. They were formed by drying the colloidal solutions in Petri dishes in the chamber containing vapor-adsorbing substances at room temperature. EPR measurements were carried out using X-band $(\sim 9.5 \mathrm{GHz}) \quad$ spectrometer "Radiopan" at room temperature.

\section{Results and discussion}

To study the variation of the NPs size with the sequential addition of the precursors to the growth solution we measured the spectral dependences of the optical density of the colloidal solutions at every step of synthesis. As was shown in [10], the position of the optical absorption edge reflects the change of the average radius of NPs in the solution, thus these measurements can provide information about the NPs size during the growth.

Fig. 2 and 3 present the experimental data on the optical density of colloidal solutions of NPs CdS and CdS:Mn at different stages of synthesis (for the details see Table). The curves for the stage 1 are not shown in the Figs 2 and 3 because the starting growth solution is transparent in the whole spectral range studied.

Based on the above experimental data, the optical bandgap $\left(E_{g}\right)$ of NPs in the solutions was estimated using the second derivative approach [11]. Fig. 4 shows the dependence of the calculated band gap of NPs on the concentration of precursors. Note that the precursor concentration value shown on the horizontal axis of the plot reflects the amount of precursors that have already took part in the chemical reaction, i.e., this concentration is equivalent to the content of $\mathrm{CdS}$ compound in the growth solution.

It is seen that with the increasing of precursors concentration the band gap of both undoped and doped NPs decreases in agreement with previous observations 
by other researchers $[12,13]$. However, while at the initial stages of synthesis (the concentration of salts $<5 \cdot 10^{-3} \mathrm{M}$ ) the band gap of doped and undoped NPs is almost the same, the further variation of the band gap is very different for the two types of NPs, and at the final stages of growth the band gap value of doped NPs is by almost $120 \mathrm{meV}$ larger as compared to undoped NPs.

To analyze the discrepancies in the band gap behavior of undoped and doped NPs, one should consider two main factors that can influence the band gap value in the NPs under investigation. These factors are: i) the confinement-induced increase of the band gap in NPs [10], ii) the complex concentration-dependent variation of the band gap of CdSMn. The former factor is peculiar for both types of NPs while the latter one influences only the band gap of doped NPs. According to the literature data on the dependence $E_{g}$ vs. manganese content in bulk CdS:Mn [14] and nanoCdS:Mn [15] the band gap of CdS:Mn changes nonmonotonously with concentration: in the range $0-4 \%$ of $\mathrm{Mn}$ the band gap value slightly decreases and in the range $4-40 \%$ the band gap increases almost by $300 \mathrm{meV}$.

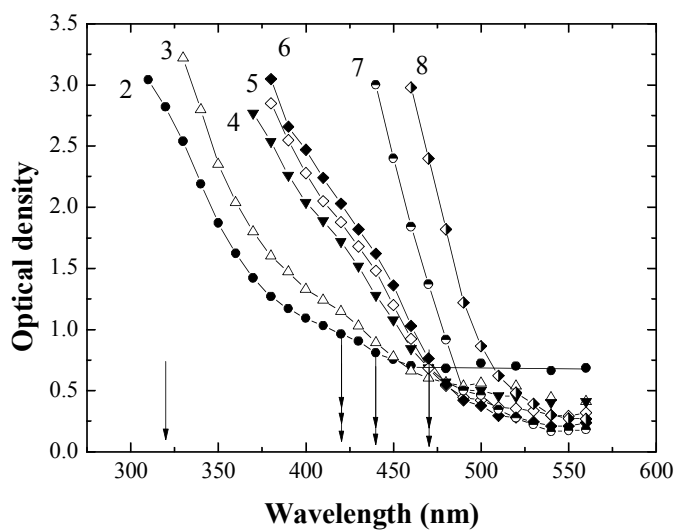

Fig. 2. Spectral dependencies of the optical density of the colloidal solution of CdS NPs measured at different stages of synthesis. Curves 2-8 correspond to the stages 2-8 listed in Table for CdS.

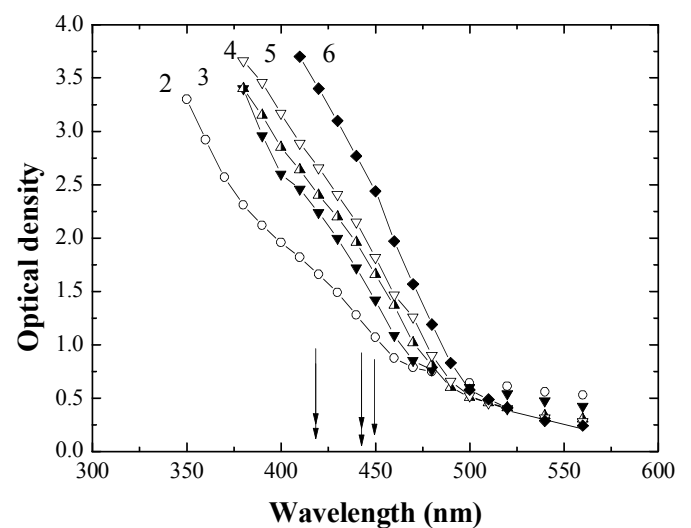

Fig. 3. Spectral dependences of the optical density measured at different stages of the synthesis of CdS:Mn nanoparticles. Curves 2-6 correspond to the stages 2-6 listed in Table for CdS:Mn.
Keeping in mind the above two factors we precede with the comparison of the band gap variation for undoped and doped NPs in course of colloid synthesis.

The equality of $E_{g}$ values of doped and undoped NPs at early stages of growth is indicative of the equivalence of the processes of nucleation in both cases. Thus one can conclude that nucleation of NPs in both growing procedures starts with the formation of undoped $\mathrm{CdS}$ seeds. This agrees with the findings of other authors [4, 16-19]. Upon further rising of the precursors concentration the distinctions in the values of the bandgap for the undoped and doped nanoparticles become more pronounced. To explain the difference in the $E_{g}$ values observed we will discuss two extreme cases when only one of the above factors is dominating.

If one assumes that size-dependent mechanism of band gap variation is dominating then one has to conclude that the presence of $\mathrm{Mn}$ in the solution strongly inhibits NPs growth. In this assumption the size of Mndoped NPs has to be about $5.5 \mathrm{~nm}$ that is smaller than the average size of undoped NPs $(\sim 6.5 \mathrm{~nm})$. Similar slowing of NPs growth due to the presence of ions of magnetic impurities was observed in [20].

In the framework of another extreme assumption one can ascribe difference in the band gap values of undoped and doped NPs solely to the influence of the Mn dopant. It means that we assume that CdSMn NPs have the same size as undoped CdS NPs and the band gap increase is totally composition-related.

According to $[14,15]$ the observed difference in $E_{g}$ that is equal to $120 \mathrm{meV}$ points to $\mathrm{Mn}$ content of more than $20 \%$. This doping level is rather high, so we have to check this assumption by some additional methods.

In order to get information about the presence of $\mathrm{Mn}^{2+}$-ions in CdS particles the measurements of EPR are a helpful tool. Fig. 5 represents EPR spectrum of studied PVA/CdS:Mn nanoparticles nanocomposite. The spectrum demonstrates a multiple peak pattern that is characteristic of the $\mathrm{Mn}^{2+}$ species. Observation of these six lines is usually assumed as a proof of $\mathrm{Mn}^{2+}$ ions presence in the nanocomposite. More detailed study of the spectrum shows that the sextet observed is asymmetric and distance between the peaks is not constant. It means that at least two Mn positions must be taken into account at the analysis.

By analogy with the bulk materials, for the description of nanoobjects the same values of hyperfine interaction constant are used in literature [21, 22]. We used three components for the fitting of our spectra. The first fitting component with hyperfine interaction constant 70 Gauss is assigned to single $\mathrm{Mn}^{2+}$-ions located in the bulk of NPs. The second component with larger hyperfine-splitting of 95 Gauss - is assigned to $\mathrm{Mn}^{2+}$-ions located in the near-surface positions in the NPs. Indeed, the $\mathrm{Mn}^{2+}$-ions that are close to the surface must be less covalently bonded. This results in a higher density of the electron wavefunction on the manganese nucleus, and thus the hyperfine interaction must be stronger. The third fitting component is the broad line 
with peak to peak splitting of 500 Gauss. The latter line originates from the regions where $\mathrm{Mn}$ ions are situated very close to each other and $\mathrm{Mn}-\mathrm{Mn}$ interactions are rather strong.

Thus, the observation of the two first components demonstrates that manganese is located both inside a NP and near its surface. Moreover, the presence of the third component shows that concentration of $\mathrm{Mn}$ is sufficiently high for manganese ions to interact. However, the overall view of the EPR spectrum evidences that the content of $\mathrm{Mn}^{2+}$ cannot exceed a couple of percents. This latter fact leads to the conclusion that the dominating effect that controls the band gap of NPs is quantum confinement. Thus, the growth of NPs in the solution containing $\mathrm{Mn}$ ions is inhibited, the size of doped NPs is smaller compared to the undoped ones and doping leads only to minor variation of the band gap.

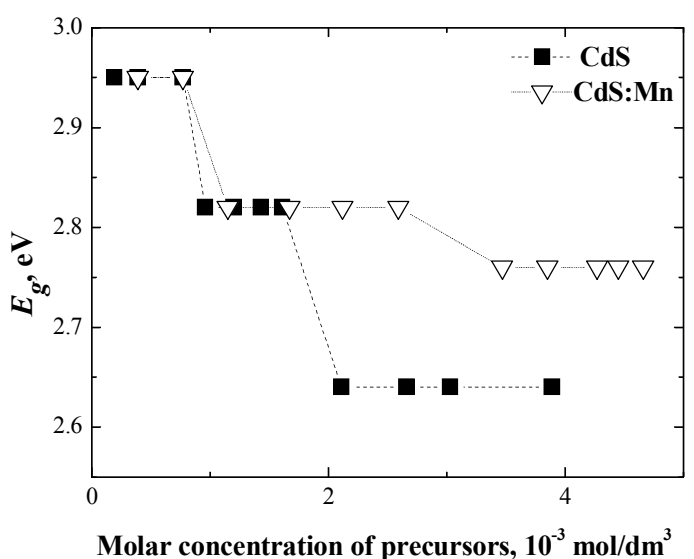

Fig. 4. Dependence $E_{g}$ of nanoparticles on the concentration of precursors of ions $\mathrm{Cd}^{2+}$ тa $\mathrm{S}^{2-}$, which have reacted completely. Solid squares and blank triangles correspond to the calculated values of $E_{g}$ for $\mathrm{CdS}$ and CdS:Mn NPs, respectively. Lines are the guides for the eye.

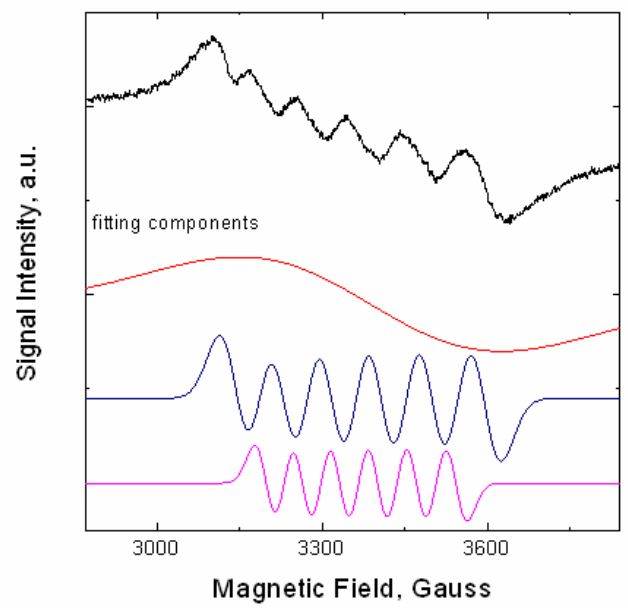

Fig. 5. EPR spectra and fitting parameters of nanocomposite PVA/CdS:Mn nanoparticles.

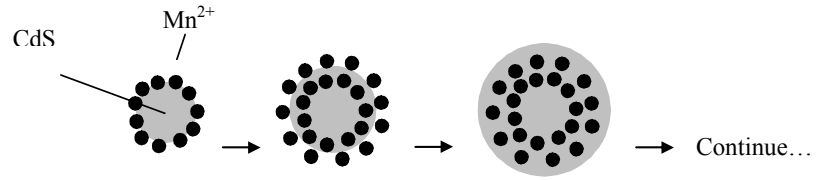

Fig. 6. A diagram of incorporating paramagnetic impurities into a nanoparticle by means of the method of adsorption doping.

The experimental results obtained make it possible to propose a model of adsorptive doping of the $\mathrm{CdS}$ nanoparticles by the $\mathrm{Mn}^{2+}$ ions which is represented in Fig. 6. The growth of NPs proceeds as follows: at the initial stages of synthesis the undoped CdS seed of a future NP is formed. The Mn ions from the solution are adsorbed on the surface of an already formed seed. The following addition of precursors to the solution leads to the increase of a NP and manganese appears to be covered with CdS. This increased NP is also covered by the adsorbed $\mathrm{Mn}$ and then with a new layer of $\mathrm{CdS}$, and so on. As a result, the doped NP consists of an undoped core and the Mn-containing periphery.

\section{Conclusion}

In the present study, we have synthesized colloidal solutions of undoped CdS and doped NPs in PVA and formed solid films of NP/polymer composite. We have studied the absorption of both types of colloidal NPs at different stages of synthesis and have demonstrated that the absorption edge and, consequently, the band gap of NPs varies differently: at early stages of synthesis the band gap of both types of NPs is the same while it differs drastically when NPs grow larger. We have also measured EPR spectra of NP/polymer composite and demonstrated that the content of $\mathrm{Mn}$ cannot be very large, not exceeding couple of percents. Thus, we have concluded that introduction of $\mathrm{Mn}$ ions into the growth solution inhibits NPs growth. The model of the adsorptive doping of CdS NPs with manganese was proposed.

\section{References}

1. X. Michalet, F.F. Pinaud, L.A. Bentolila, J.M. Tsay, S. Doose, J.J. Li, G. Sundaresan, A.M. Wu, S.S. Gambhir, S. Weiss, Quantum dots for live cells, in vivo imaging, and diagnostics // Science, 307, p. 538-544 (2005).

2. Y.-J. Doh, K.N. Maher, L. Ouyang, C.L. Yu, H. Park, J. Park, Electrically driven light emission from individual CdSe nanowires // Nano Lett. 8, p. $4552-4556$ (2008).

3. R. Beaulac, P.I. Archer, S.T. Ochsenbein, D.R. Gamelin, $\mathrm{Mn}^{2+}$-doped CdSe quantum dots: New inorganic materials for spin-electronics and 
spin-photonics // Adv. Funct. Mater. 18, p. 38733891 (2008)

4. D.J. Norris, A.L. Efros, S.C. Erwin, Doped nanocrystals // Science, 319, p.1776-1779 (2008).

5. C.-W. Wang, M.G. Moffitt, Nonlithographic hierarchical patterning of semiconducting nanoparticles via polymer/polymer phase separation // Chem. Mater. 17, p. 3871-3878 (2005).

6. G. Kickelbick, Hybrid Materials: Synthesis, Characterization, and Applications. Wiley-VCH Verlag GmbH \& Co. KGaA, 2007.

7. T. Di Luccio, A. Maria Laera, L. Tapfer, S. Kempter, R. Kraus, B. Nickel, Controlled nucleation and growth of $\mathrm{CdS}$ nanoparticles in a polymer matrix // J. Phys. Chem. B, 110, p. 1260312609 (2006).

8. J.X. Yao, G.L. Zhao, D. Wang, G.R. Han, Solvothermal synthesis and characterization of $\mathrm{CdS}$ nanowires/PVA composite films // Mater. Lett. 59, p. 3652-3655 (2005).

9. H. Yang, S. Santra, P. H. Holloway, Syntheses and applications of Mn-doped II-VI semiconductor nanocrystals // J. Nanosci. and Nanotechnol. 5, p. 1364-1375 (2005).

10. L.E. Brus, Simple model for the ionization potential, electron affinity, aqueous redox potentials of small semiconductor crystallites // J. Chem. Phys. 79, p. 5566-5571 (1983).

11. A.I. Ekimov, F. Hache, M.C. Schanne-Klein, D. Ricard, C. Flytzanis, I.A. Kudryavtsev, T.V. Yazeva, A.V Rodina, Absorption and intensity-dependent photoluminescence measurements on CdSe quantum dots: assignment of the first electronic transitions // J. Opt. Soc. Am. $B$, 10, p. 100-107 (1993).

12. D.S. Yoo, S.Y. Ha, I.G. Kim, M.S. Choo, K.W. Kim, E.S. Lee, Characterization of $\mathrm{CdS}$ nanoparticles embedded in polyvinyl alcohol // New Physics: Sae Mulli (The Korean Physical Society) 61, p. 680-686 (2011).
13. M. Pattabi, B. Saraswathi Amma, K. Manzoor, Photoluminescence study of PVP capped CdS nanoparticles embedded in PVA matrix // Mater. Res. Bull. 42, p. 828-835 (2007).

14. J.K. Furdyna, Diluted magnetic semiconductors // J. Appl. Phys. 64, p. R29-R64 (1988).

15. L. Levy, N. Feltin, D. Ingert, M.P. Pileni, Three dimensionally diluted magnetic semiconductor clusters $\mathrm{Cd}_{1-\mathrm{y}} \mathrm{Mn}_{\mathrm{y}} \mathrm{S}$ with a range of sizes and compositions: Dependence of spectroscopic properties on the synthesis mode // J. Phys. Chem. $B$, 101, p. 9153-9160 (1997).

16. G.M. Dalpian, J.R. Chelikowsky, Self-purification in semiconductor nanocrystals // Phys. Rev. Lett. 96, 226802 (2006).

17. G.M. Dalpian, J.R. Chelikowsky, Dalpian and Chelikowsky Reply // Phys. Rev. Lett. 100, 179703 (2008).

18. M.H. Du, S.C. Erwin, A.L. Efros, D.J. Norris, Comment on "Self-Purification in Semiconductor Nanocrystals" // Phys. Rev. Lett. 100, 179702 (2008).

19. S.C. Erwin, L. Zu, M.I. Haftel, A.L. Efros, T.A. Kennedy, D.J. Norris, Doping semiconductor nanocrystals // Nature, 436, p. 91-94 (2005).

20. J.D. Bryan, D.A. Schwartz, D.R. Gamelin, The influence of dopants on the nucleation of semiconductor nanocrystals from homogeneous solution // J. Nanosci. and Nanotechnol. 5, p. 14721479 (2005).

21. J.D. Bryan, D.R. Gamelin, Doped semiconductor nanocrystals: Synthesis, characterization, physical properties, and applications // Progr. in Inorgan. Chem. 54, p. 47-126 (2005).

22. C. Barglik-Chory, C. Remenyi, C. Dem, M. Schmitt, W. Kiefer, C. Gould, C. Ruster, G. Schmidt, D.M. Hofmann, D. Pfistererd, G. Muller, Synthesis and characterization of manganese-doped CdS nanoparticles // Phys. Chem. Chem. Phys. 5, p. 1639-1643 (2003). 